\title{
$\omega$-3 PUFAs and Resveratrol Differently Modulate Acute and Chronic Inflammatory Processes
}

\author{
Joseph Schwager, ${ }^{1}$ Nathalie Richard, ${ }^{1}$ Christoph Riegger, ${ }^{1}$ and Norman Salem Jr. ${ }^{2}$ \\ ${ }^{1}$ DSM Nutritional Products Ltd., Department of Human Nutrition \& Health, P.O. Box 2676, 4002 Basel, Switzerland \\ ${ }^{2}$ DSM Nutritional Products Ltd., 6480 Dobbin Road, Columbia, MD 21045, USA \\ Correspondence should be addressed to Joseph Schwager; joseph.schwager@dsm.com
}

Received 27 November 2014; Accepted 15 April 2015

Academic Editor: Hui-Min Su

Copyright (c) 2015 Joseph Schwager et al. This is an open access article distributed under the Creative Commons Attribution License, which permits unrestricted use, distribution, and reproduction in any medium, provided the original work is properly cited.

$\omega$-3 PUFAs and polyphenols have multiple effects on inflammation in vivo and in vitro. The effects of eicosapentaenoic acid (EPA), docosahexaenoic acid (DHA), and resveratrol (RV) were investigated in LPS-stimulated peripheral blood leukocytes (PBLs) (i.e., acute inflammation) and IL-1 $\beta$ activated human chondrocytes (i.e., chronic inflammation). Inflammatory mediators including chemokines, cytokines, interleukins, and $\mathrm{PGE}_{2}$ were measured by multiplex analysis and gene expression was quantified by RTPCR. In PBLs, RV decreased the secretion of PGE 2 , CCL5/RANTES, and CXCL8/IL-8 but increased IL-1 $\beta$, IL-6, and IL-10. In contrast to RV, $\omega$-3 PUFAs augmented the production of PGE 2 and CXCL8/IL-8. EPA and DHA similarly affected the pattern of inflammatory mediators. Combination of RV and $\omega$-3 PUFAs exerted synergistic effects on CCL5/RANTES and had additive effects on IL-6 or CXCL8/IL-8. Both $\omega$-3 PUFAs and RV reduced catabolic gene expression (e.g., MMPs, ADAMTS-4, IL-1 $\beta$, and IL-6) in activated chondrocytes. The data suggest that $\omega$-3 PUFAs and RV differ in the regulation of acute inflammation of peripheral blood leukocytes but have common properties in modulating features related to chronic inflammation of chondrocytes.

\section{Introduction}

Cells and tissues respond to changes in physiological milieu as well as to external insults. Acute inflammatory processes are part of the normal response of the organism and are indispensable to restoring homeostasis. This is achieved by multiple mediators that initiate and modulate the extent and duration of and also resolve inflammatory processes. When these feedback mechanisms fail or are dysregulated, inflammatory mediators might remain in a status of low-grade or chronic inflammation as observed in obesity, cardiovascular diseases (CVD), diabetes, and arthritis. Cells of the immune system including peripheral blood leukocytes (PBLs) have a key role in the regulation of acute and chronic inflammation. They are continuously exposed to various nutrients, which can therefore influence their metabolic and functional status. Food components like $\omega$-3 polyunsaturated acids (PUFAs) or micronutrients such as vitamins and antioxidants were found to beneficially modulate inflammatory processes and diseases [1-3]. Specifically, eicosapentaenoic acid (EPA) and docosahexaenoic acid (DHA) modulate eicosanoid metabolism and are precursors of mediators that are essential for the resolution of inflammation [4]. The anti-inflammatory effects of $\omega$-3 PUFAs have been demonstrated in cellular systems and human studies [5-16] (see also [17] for review). On the other hand, in vitro activated chondrocytes are an adequate cellular model to analyse potential effects of nutrients on cells such as chondrocytes at conditions of chronic inflammation.

In this study we investigated the effects of $\omega-3$ PUFAs on a panel of inflammatory mediators (cytokines, interleukins, and chemokines) produced by activated PBL from healthy individuals and by stimulated human chondrocytes and compared them to the natural phenolic compound resveratrol (RV) that has multiple effects on the inflammatory response [18]. We show that the substances have distinct effects on leukocytes and chondrocytes, which reflect acute and chronic inflammatory processes, respectively.

\section{Materials and Methods}

2.1. PUFAs, Resveratrol, and Cell Culture Reagents. Free fatty acid eicosapentaenoic acid (EPA), docosahexaenoic acid 
(DHA), and resveratrol were from Sigma/Aldrich (Saint Louis, MO). E. coli lipopolysaccharide (LPS, serotype 055:B5) and fetal bovine serum (FBS) were from Sigma/Aldrich (Saint Louis, MO). Cell culture reagents (RPMI 1640, 2-mercaptoethanol, and MEM nonessential amino acids (NEAA)) were from Invitrogen (Carlsbad, CA). IL-1 $\beta$ was purchased from PeproTech EC (London, UK). Substances were dissolved in DMSO and added to cells shortly before treating the cells with biological stimulators. Final concentrations of PUFAs and resveratrol were $10-50 \mu \mathrm{mol} / \mathrm{L}$ and $1.25-$ $50 \mu \mathrm{mol} / \mathrm{L}$, respectively.

2.2. Cell Culture. Human peripheral blood leukocytes (PBLs) were isolated from buffy coats obtained from healthy individuals and treated with inflammatory stimuli as described [19]. Briefly, PBLs were cultured in phenol red-free RPMI 1640 (containing $0.25 \% \mathrm{FBS}, 0.1 \mathrm{mM}$ NEAA, $50 \mathrm{U} / \mathrm{mL}$ penicillin, and $50 \mu \mathrm{mg} / \mathrm{mL}$ streptomycin) and stimulated with $1 \mu \mathrm{g} / \mathrm{mL}$ LPS in the presence of graded amounts of substances. Human in vitro PBL experiments were approved by the Swiss Federal Office of Public Health (number A050573/2) and the Ethical Commission of the Kanton Aargau, Switzerland. Cells were lysed in RLT buffer (Qiagen, Hilden, Germany) after $12 \mathrm{~h}$ of culture and total RNA was extracted. Alternatively, cells were cultured for $24 \mathrm{~h}$; and secreted mediators were analyzed in culture supernatants.

Normal human articular chondrocytes from knee (NHAC-kn), prepared from different individuals, were cultured in chondrocyte growth medium (Lonza, Walkersville, MD) and used for experiments between passages 3 and 6 [20]. Cells $\left(0.5 \times 10^{6}\right.$ per well $)$ were seeded into 6 -well plates and confluent cells were activated with IL-1 $\beta(10 \mathrm{ng} / \mathrm{mL})$ in the presence of graded concentrations of test substances for 4-24h [20].

2.3. RNA Isolation, cDNA Synthesis, and RT-PCR. The isolation of total RNA, synthesis of CDNA, and quantitative RTPCR have been detailed previously $[19,20]$.

2.4. Multiparametric Analysis of Cytokines, Chemokines, and Interleukins. Multiparametric kits were purchased from BioRad Laboratories (Hercules, CA) and used in the LiquiChip Workstation IS 200 (Qiagen, Hilden, Germany) to measure the amount of secreted proteins. Data evaluation was done using the LiquiChip Analyser software (Qiagen). $\mathrm{PGE}_{2}$ were measured as described previously [20].

2.5. Statistical Analysis. Data were evaluated by statistical tools described previously [20]. A $p$ value $<0.05$ (calculated by using Student's $t$-test or one-way ANOVA) was considered to reflect statistically significant differences.

2.6. Calculation of Combination Effects. The algorithm developed by Chou and Talalay has been used to calculate synergism of inhibitory effects $[21,22]$. Interactions were quantified by the combination index (CI) as described by Pappa et al. [23]: Using CalcuSyn software (Biosoft, Ferguson, MO), a CI was computed for every fraction affected. CI $<1$ reflects synergistic inhibition of the respective inflammatory parameter; if $\mathrm{CI}=1$ the substances have additive interactions; when $\mathrm{CI}>$ 1 the interaction of substances reflects antagonism.

\section{Results}

3.1. Resveratrol and $\omega-3$ PUFAs Modulate the Production of Cytokines and Chemokines in Leukocytes. In order to investigate the effects of nutrients on acute inflammatory responses, PBLs from different individuals were activated with LPS in the presence of graded amounts of substances. LPS triggered substantial secretion of cytokines, chemokines, and $\mathrm{PGE}_{2}$; some of these differed considerably between individuals with respect to IL- $1 \beta$, TNF- $\alpha$, and CCL5/RANTES showing the largest interindividual variations (Table 1). The effects of resveratrol (RV) and $\omega-3$ PUFAs on the inflammatory mediators of activated PBLs are shown in Figure 1. In order to correct for interindividual variations, the data are expressed as a ratio [(substance + LPS-treated cells)/LPS-treated cells]. $\mathrm{RV}$ drastically reduced the secretion of $\mathrm{PGE}_{2}$, which is dependent on the LPS-induced expression of COX-2 in monocytes/macrophages (Table 1). Conversely, interleukin- (IL-) $1 \beta$, IL- 6 , and the anti-inflammatory IL-10 were increased in the presence of RV $(6.25-25 \mu \mathrm{mol} / \mathrm{L})$. We further investigated the impact of RV on chemokine secretion. CCL5/RANTES, which recruits activated T lymphocytes [24], was augmented by high concentrations of RV $(25 \mu \mathrm{mol} / \mathrm{L})$. The neutrophil recruiting chemokine CXCL8/IL-8 was blunted by increasing RV concentration. CCL2/MCP-1, which is involved in targeted migration of resident monocytes [24] and macrophage polarization [25], was not significantly altered. In contrast, RV enhanced production of CCL4/MIP-1 $\beta$, a chemokine for subtypes of monocytes [26].

DHA modulated the production of inflammatory mediators of LPS-activated leukocytes in a different manner (Figure 1). It enhanced $\mathrm{PGE}_{2}$ production (at 5-20 $\mu \mathrm{mol} / \mathrm{L}$ ). IL- $1 \beta$ and IL- 6 were produced to larger extents when DHA was included in the cellular assay. Similarly, DHA markedly enhanced CXCL8/IL-8 secretion, whereas it mitigated CCL5/ RANTES. The production of CCL2/MCP-1 and CCL4/MIP$1 \beta$ by activated leukocytes was only influenced by high concentrations of DHA. EPA shared with DHA a similar activity pattern on the production of inflammatory mediators. It should be noted that the extent of the response of PBLs to LPS was subject to large interindividual variations that presumably mirrored the differing immune status of the donors (Table 1).

3.2. Altered Gene Expression in Activated PBLs. By using quantitative RT-PCR, we investigated the impact of the substances on the transcription of inflammatory genes in PBLs after $12 \mathrm{~h}$ of LPS-stimulation, when many LPS-responsive genes were still upregulated $[19,27]$. RV had only a minor influence on IL-1 $\beta$ mRNA levels, whereas it significantly augmented IL-6 transcription (Figure 2), consistent with the increased IL-6 secretion (Figure 1). Similarly, IL-10 mRNA levels of RV-treated cells matched the higher secretion of 
TABLE 1: Secreted proteins of unstimulated and stimulated PBLs obtained from different subjects.

\begin{tabular}{|c|c|c|c|c|c|}
\hline Parameter & $\begin{array}{l}\text { Unstimulated cells } \\
(\mathrm{pg} / \mathrm{mL})\end{array}$ & $\begin{array}{l}\text { LPS-stimulated cells } \\
(\mathrm{pg} / \mathrm{mL})\end{array}$ & $\begin{array}{c}\text { Range } \\
(\mathrm{pg} / \mathrm{mL})\end{array}$ & ${ }^{(2)} R=\left(\mathrm{RV}^{(3)}+\mathrm{LPS}\right) / \mathrm{LPS}$ & ${ }^{(2)} R=\left(\mathrm{EPA}^{(3)}+\mathrm{LPS}\right) / \mathrm{LPS}$ \\
\hline IL-1 $\beta$ & $0 \pm 0^{(1)}$ & $7889 \pm 1250^{(1)}$ & $2025-18000$ & $2.62 \pm 0.30$ & $2.76 \pm 0.94$ \\
\hline IL-6 & $1 \pm 1$ & $111644 \pm 19519$ & $51300-209000$ & $1.76 \pm 0.30$ & $2.29 \pm 0.51$ \\
\hline IL-10 & $1 \pm 1$ & $1279 \pm 230$ & $388-2530$ & $1.29 \pm 0.35$ & $1.06 \pm 0.21$ \\
\hline IL-12 (p70) & $0 \pm 0$ & $14 \pm 7$ & $0-73$ & - & - \\
\hline TNF- $\alpha$ & $0 \pm 0$ & $3499 \pm 1300$ & $418-12750$ & $0.86 \pm 0.15$ & $1.44 \pm 0.65$ \\
\hline CCL2/MCP-1 & $1 \pm 1$ & $724 \pm 163$ & $363-2065$ & $0.77 \pm 0.26$ & $0.95 \pm 0.14$ \\
\hline CCL3/MIP-1 $\alpha$ & $1 \pm 1$ & $14718 \pm 2698$ & $2720-28250$ & $1.51 \pm 0.56$ & $1.27 \pm 0.47$ \\
\hline CCL4/MIP-1 $\beta$ & $182 \pm 31$ & $68950 \pm 12844$ & $33300-162500$ & $1.75 \pm 0.46$ & $0.81 \pm 0.17$ \\
\hline CCL5/RANTES & $536 \pm 117$ & $6013 \pm 1978$ & $670-20300$ & $1.55 \pm 1.57$ & $0.77 \pm 0.14$ \\
\hline CCL11/Eotaxin & $28 \pm 8$ & $448 \pm 82$ & $78-878$ & $1.16 \pm 0.11$ & $1.04 \pm 0.09$ \\
\hline CXCL8/IL-8 & $311 \pm 35$ & $298980 \pm 50712$ & $108800-614000$ & $0.79 \pm 0.35$ & $2.10 \pm 0.60$ \\
\hline $\mathrm{PGE}_{2}$ & $121 \pm 15$ & $3437 \pm 499$ & $1680-6249$ & $0.11 \pm 0.17$ & $2.10 \pm 0.64$ \\
\hline
\end{tabular}

${ }^{(1)}$ Mean \pm SEM of values obtained from PBLs of 8 different subjects (each done in duplicate).

${ }^{(2)}$ Ratio: (metabolites produced by PBLs treated with substance + LPS)/metabolites produced by PBLs treated with LPS. Mean \pm SD of triplicate values obtained from PBLs of 4 different subjects.

(3) $25 \mu \mathrm{mol} / \mathrm{L} \mathrm{RV}, 20 \mu \mathrm{mol} / \mathrm{L} \mathrm{EPA}$.

IL-10. We observed a significant decrease of CXCL8/IL8 expression by RV (at $25 \mu \mathrm{mol} / \mathrm{L}$ ) (Figure 2). Conversely, CCL5/RANTES gene expression was not induced by LPSstimulation nor changed by concomitant RV treatment. DHA altered gene expression patterns in a similar way, as it changed the secretion of the respective proteins: IL- 6 expression and, to a lesser extent, IL-1 $\beta$ and IL-10 expression were augmented by DHA (at $20 \mu \mathrm{mol} / \mathrm{L}$ ) (Figure 2). CXCL8/IL-8 expression was enhanced, when DHA was included at $20 \mu \mathrm{mol} / \mathrm{L}$ in the assay. Collectively, the data indicate that RV and $\omega$-3 PUFAs regulate cytokine and chemokine production at the level of transcription.

\subsection{Effects of Combinations of $\omega-3$ PUFAs and RV Measured} in PBLs. Since we observed similar and opposite effects of substances, we investigated the pattern on inflammatory parameters produced when PBLs were treated with a combination of substances. To this aim, cells were activated in the presence of different concentrations and ratios of individual substances and the secreted mediators determined (Figure 3). $\mathrm{PGE}_{2}$ production was dominated by the inhibitory effect of $\mathrm{RV}$, which partially counterbalanced the enhancing effect of DHA (Figure 3). CXCL8/IL-8 production was controlled by DHA, since the combined treatment with RV did not result in an intermediate production. Combinations of RV and DHA synergistically inhibited CCL5/RANTES secretion, as computed by the Chou-Talalay algorithm (Figure 3). Both RV and DHA concentration-dependently enhanced IL-6 secretion and combinations thereof had additive effects. IL-1 $\beta$, however, appeared to be synergistically enhanced by RV and DHA, since the effect of combined substances largely exceeded the sum of RV and DHA applied individually.

3.4. Effects of $\omega-3$ PUFAs and RV on IL-1 $\beta$ Activated Chondrocytes. Following activation with IL-1 $\beta$, human chondrocytes (normal human articulocytes from knee, NHAC-kn) expressed various pathophysiological markers of osteoarthritis $(\mathrm{OA})$ and enzymes that degrade the extracellular matrix (ECM) [28-30]. We treated normal human chondrocytes with IL-1 $\beta$, the pathophysiological inducer of OA, and investigated the effect of RV and PUFAs on biological markers of OA. MMPs and ADAMTS, interleukins, and chemokines were upregulated in IL-1 $\beta$ treated chondrocytes [20]. In the presence of $\omega$-3 PUFAs or resveratrol, gene expression of OA markers was significantly altered (Figure 4 ). Both substances had no significant impact on gene expression of unstimulated NHAC-kn (not shown). $\omega$-3 PUFAs markedly blunted IL$1 \beta$ and IL-6 expression in stimulated NHAC-kn cells. In general, EPA and DHA induced similar alterations of gene expression in IL-1 $\beta$ activated chondrocytes. Also, MMP-3 and ADAMTS- 4 gene expression was mitigated by EPA or DHA, whereas MMP-1 expression was unaltered. MMP-13 was only slightly upregulated in IL-1 $\beta$ treated chondrocytes. Under these conditions, $\omega$-3 PUFAs had no significant impact on MMP-13 mRNA levels. Conversely, expression of CCL5/ RANTES and CXCL8/IL-8 was drastically upregulated in activated NHAC-kn cells. Concomitant treatment with EPA or DHA led to a mitigated expression of CCL5/RANTES, but they had no effect on CXCL8/IL-8 (Figure 4). EPA positively influenced chondrocyte anabolism, since the expression of Col2A and therefore the synthesis of ECM elements were increased. RV substantially altered the expression of MMP3 and ADAMTS- 4 in IL- $1 \beta$-activated NHAC, whereas its impact on chemokine gene expression was not significant. Therefore, in comparison with $\omega-3$ PUFAs, RV is predicted to have a limited impact on cartilage erosion.

\section{Discussion}

In this study we investigated whether dietary constituents altered the in vitro inflammatory response of human leukocytes from healthy individuals. RV was anti-inflammatory, 

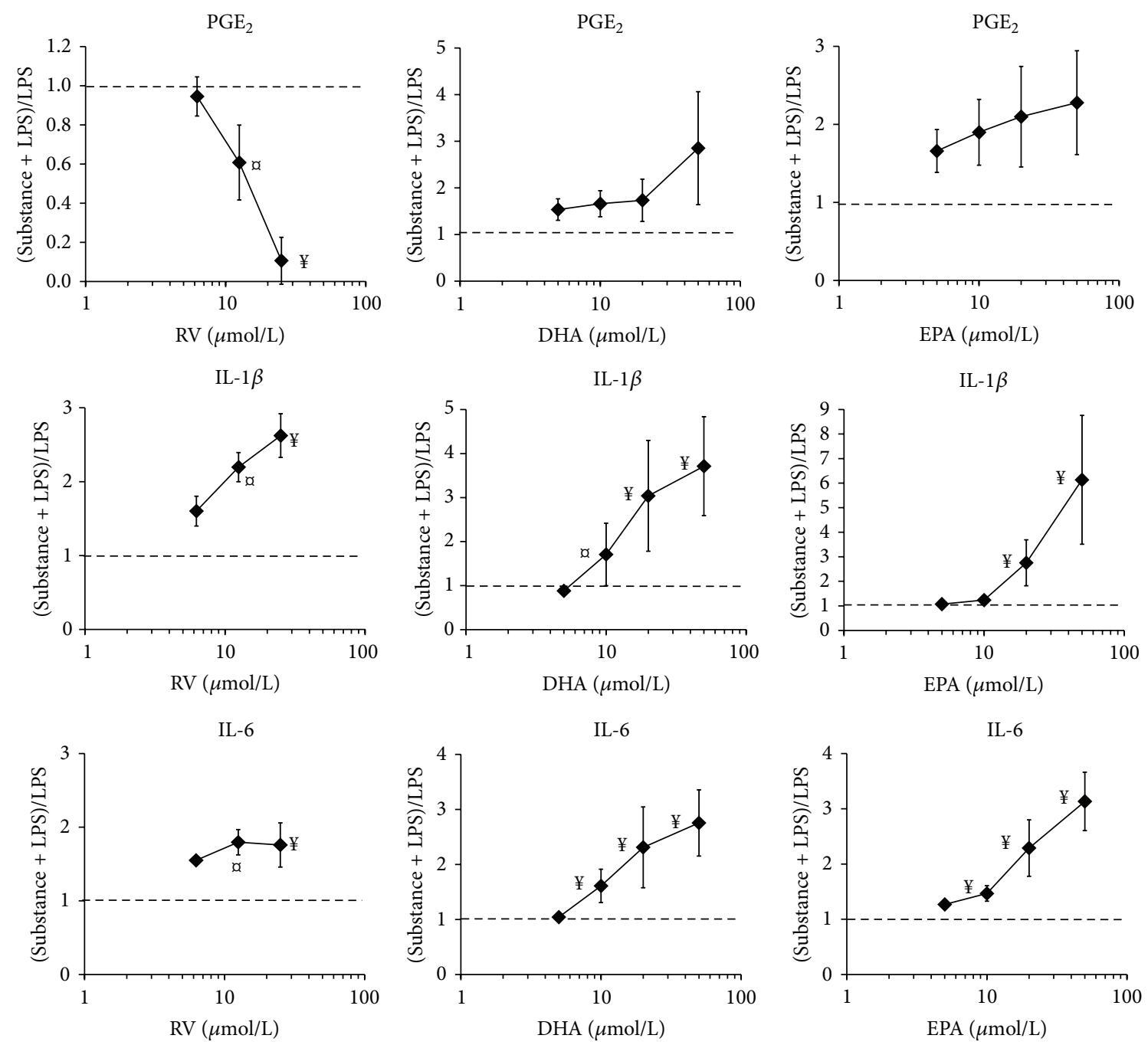

IL-10

IL-10
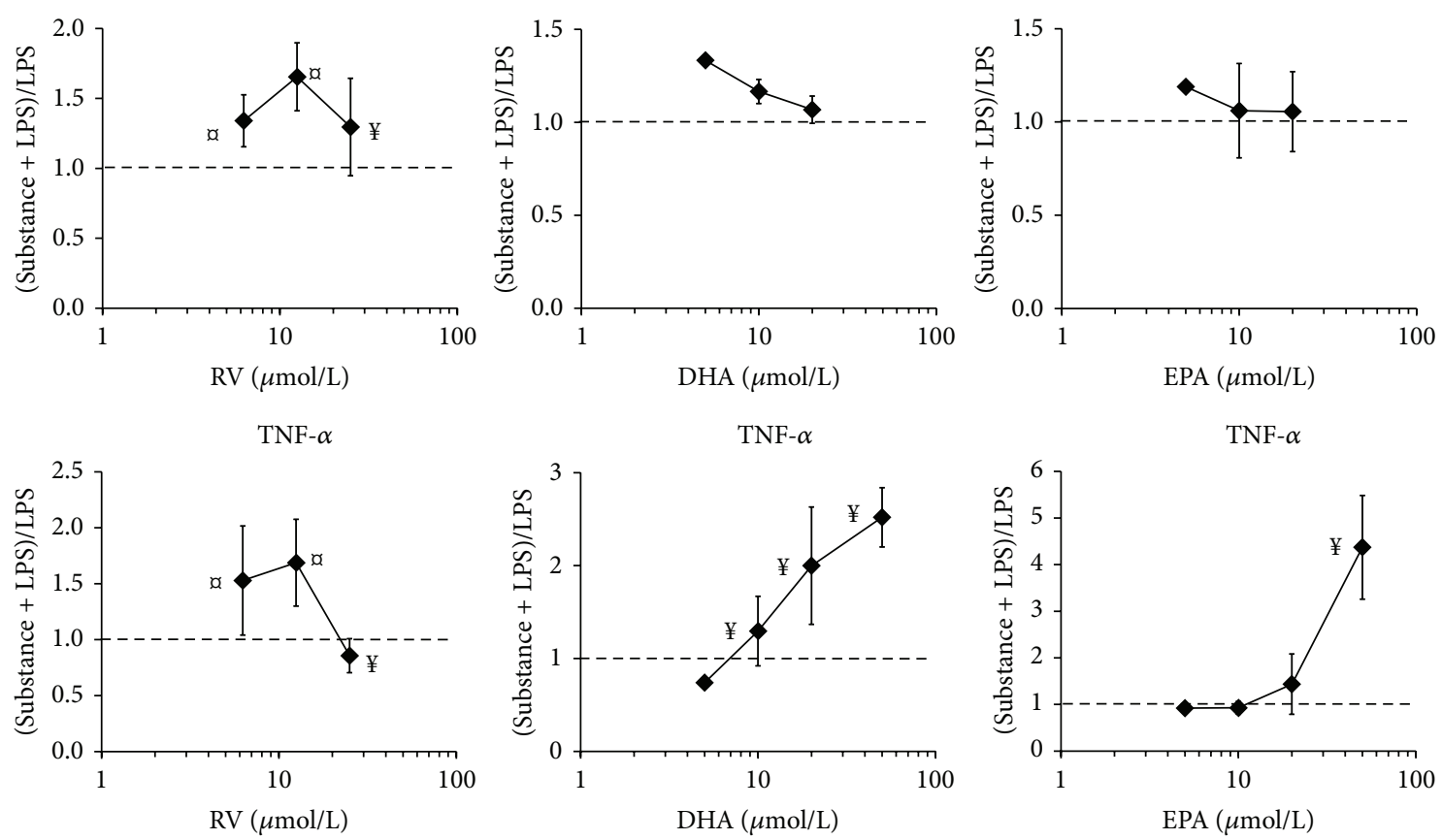

Figure 1: Continued. 

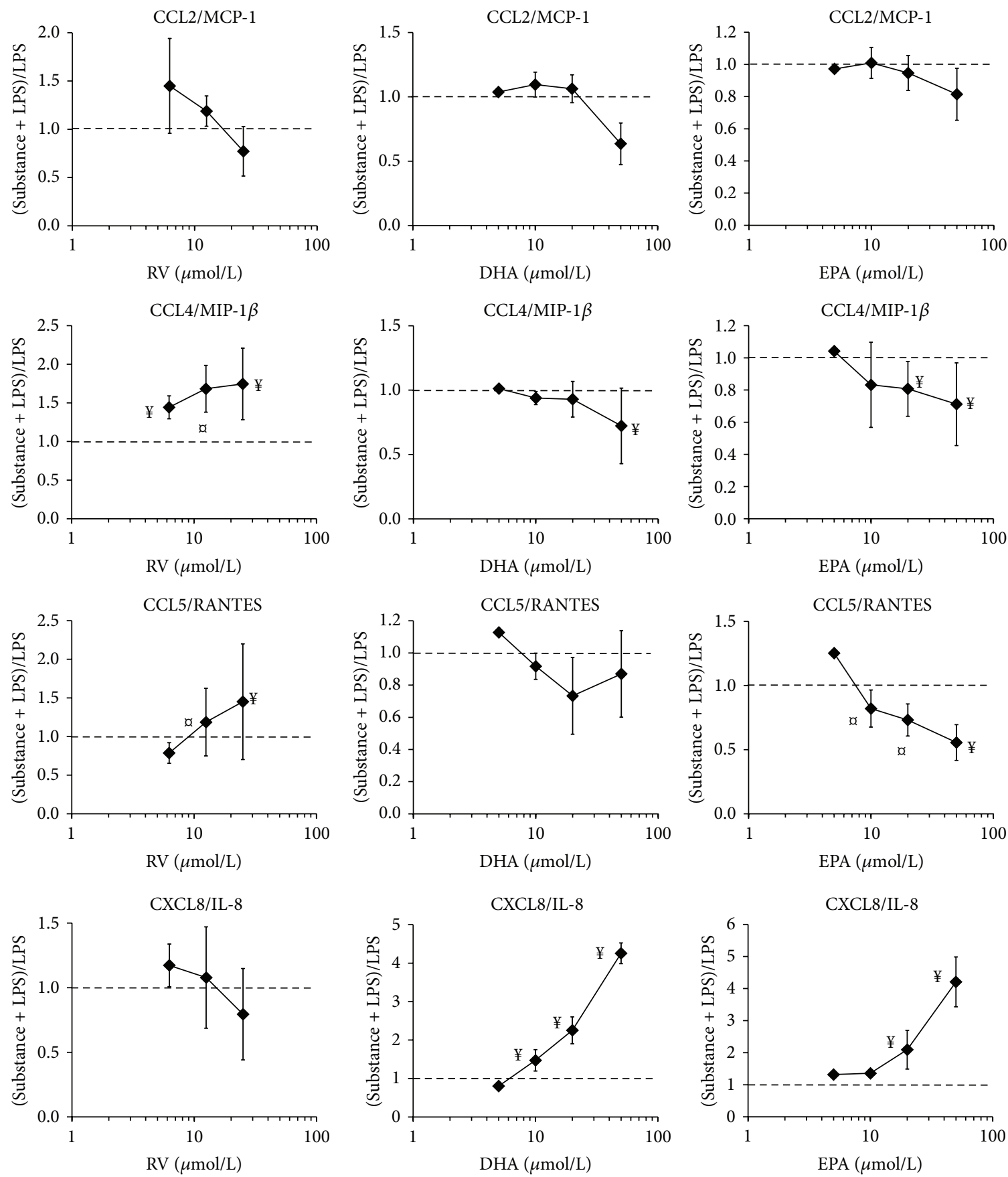

FIGURE 1: Production of inflammatory mediators by activated PBLs and its modulation by RV, DHA, and EPA. PBLs from healthy individuals were activated with LPS in the presence of the indicated substances (in $\mu \mathrm{mol} / \mathrm{L}$ ) and the inflammatory mediators in supernatants of $24 \mathrm{~h}$ cultures were determined in duplicate. The results are expressed as the ratio of substance + LPS-treated cells/LPS-treated cells. Data are given as mean \pm SD of experiments obtained from PBL from at least four individuals. The dotted line (at ratio 1) indicates the no-effect level. $x$, $¥$ indicate statistically significant differences observed in $50 \%$ and $>75 \%$ of the donors, respectively.

since it reduced, for instance, $\mathrm{PGE}_{2}$ and nitric oxide in human PBL [19] and macrophage cell lines. Yet, it had opposite effects on IL-6 produced by human PBL $[9,19,31,32]$. RV and $\omega-3$ PUFAs had similar effects on the production of IL-1 $\beta$ and IL- 6 but markedly differed in the impact on, for example, CCL2/MCP-1, CCL4/MIP-1 $\beta$, or PGE 2 production. $\omega-3$ PUFAs induced striking changes in eicosanoid metabolites
[33], modulated the inflammatory response in human PBLs via PPAR and NF- $\kappa$ B pathways $[2,8,15,34-36]$, and orchestrated the resolution of inflammation [4]. Since EPA and DHA are precursors of resolvins, they might accelerate the resolution of inflammation. The present in vitro study is the first report where effects of $\omega$-3 PUFAs on a large panel of chemokines were investigated. EPA or DHA markedly 

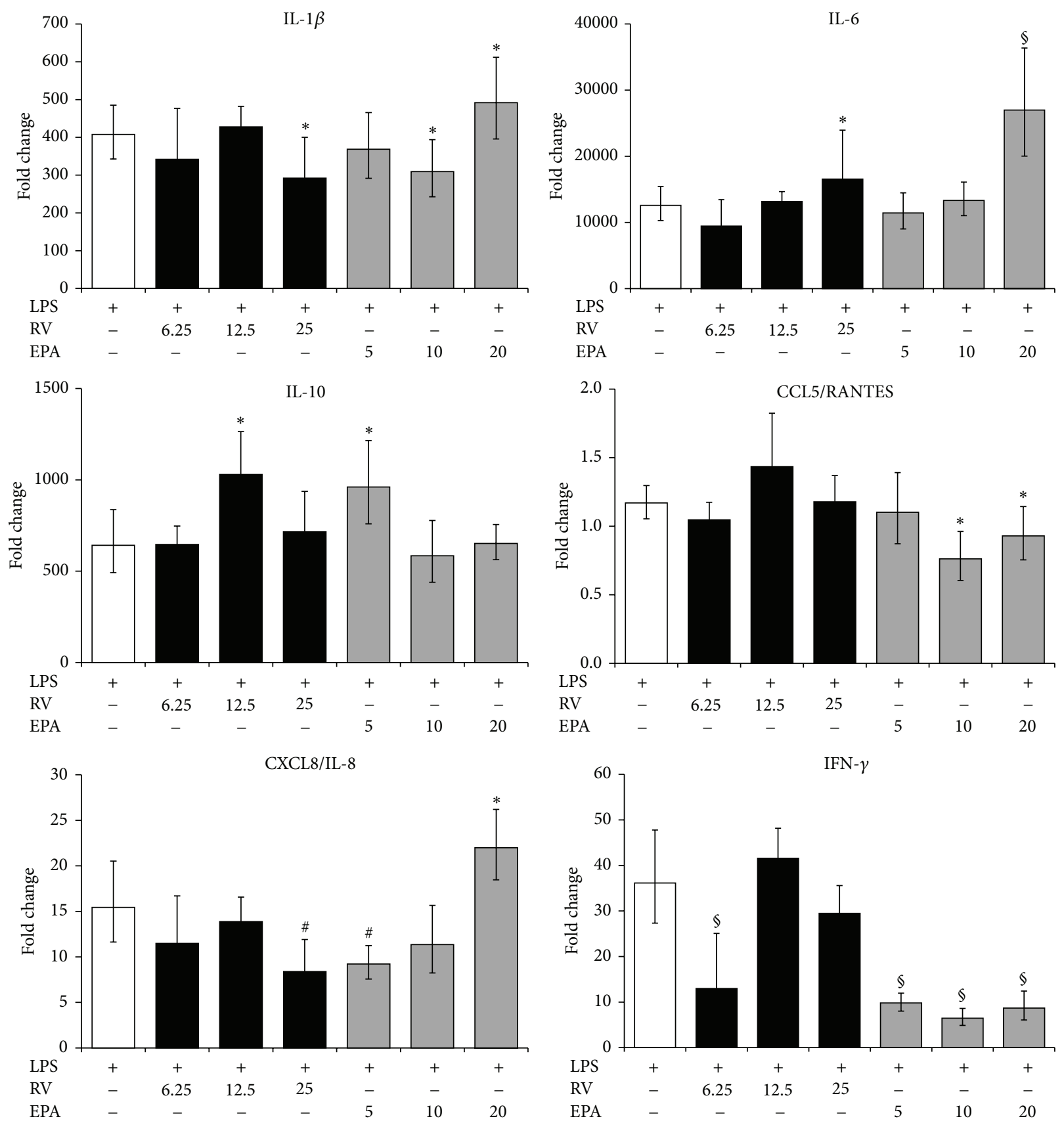

FIGURE 2: Expression of inflammatory genes in activated PBLs. Cells were activated with LPS with or without the indicated substances for $12 \mathrm{~h}$. Gene expression was quantified by RT-PCR and the data expressed as fold change compared to levels observed in unstimulated cells. Mean \pm standard errors of duplicates from three individuals are given. ${ }^{*} p<0.05,{ }^{\#} p<0.01$, and ${ }^{\S} p<0.001$ (LPS only versus LPS + substance).

increased CXCL8/IL-8 but blunted the secretion of CCL5/ RANTES. Plausibly, $\omega-3$ PUFA might increase neutrophil recruitment in the early inflammatory phase and attenuate migration of activated $\mathrm{T}$ lymphocytes during resolution. Previous studies showed modest effects of $\omega$-3 PUFAs on inflammatory markers $[37,38]$. A 12 -week fish-oil supplementation did not significantly affect plasma cytokine and chemokine concentrations, although an overall trend for an increase of inflammatory markers was observed [39] and gene expression profiles of chemokines and cytokines in peripheral blood mononuclear cells (PBMC) were affected [5, 40]. DHA levels in PBMC were inversely related to IL- $1 \beta$ and IL- 6 production [13] and diminished IL- $1 \beta$ and TNF- $\alpha$ secretion by activated PBLs in vitro [41]. Incubation of unstimulated PBLs with EPA upregulated the expression of IL-6 and CXCL8/IL-8 [42]. Since in the present study $\omega$-3 PUFAs were added shortly before the stimulation of PBLs, they might alter the release of cellular arachidonic acid and the subsequent production of eicosanoid metabolites like $\mathrm{PGE}_{2}$ and anti-inflammatory prostaglandins like $\mathrm{PGD}_{2}$. These results 

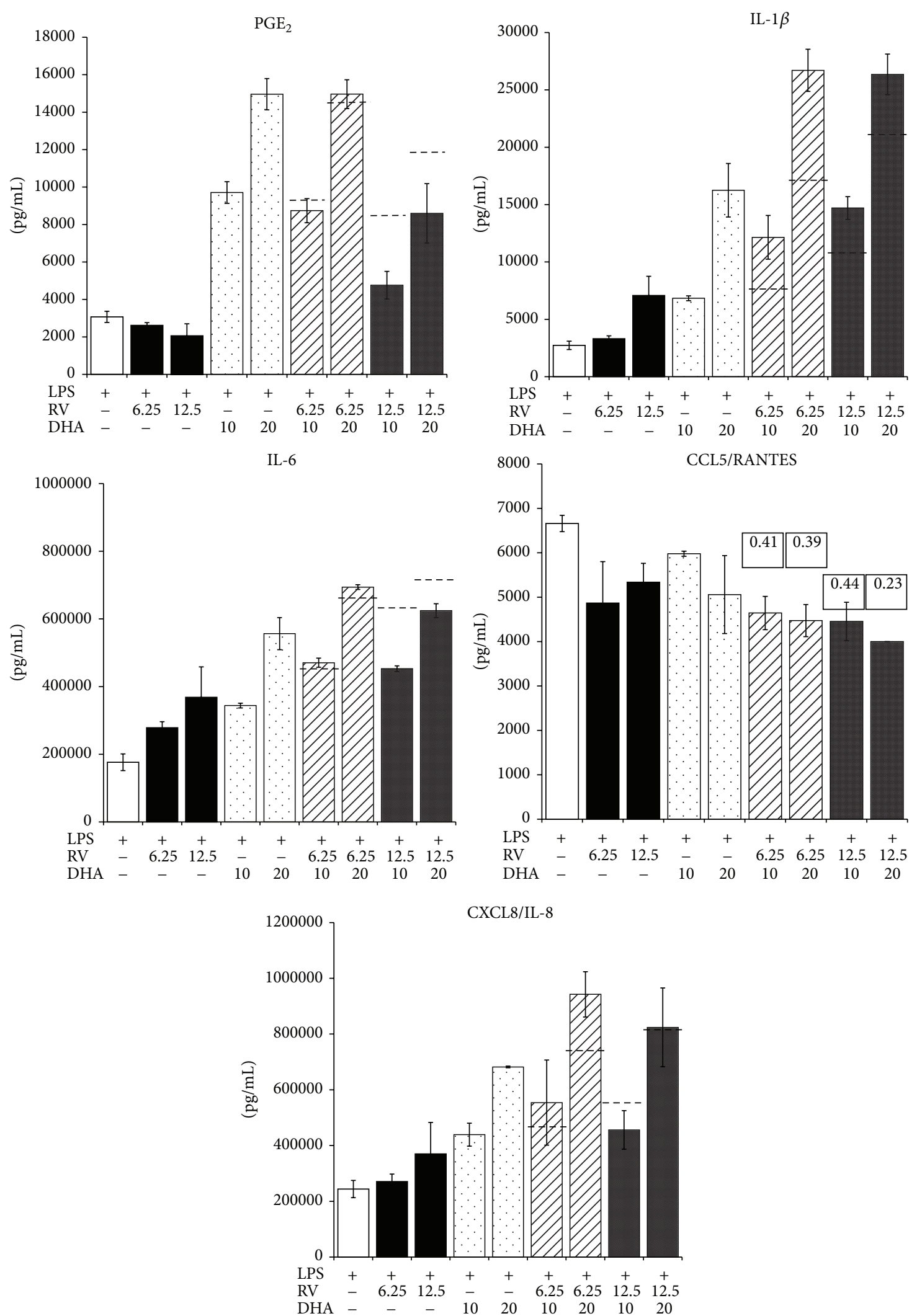

FIGURE 3: Effect of combinations of substances on inflammatory mediators produced by activated PBLs. PBLs were cultured for $24 \mathrm{~h}$ with or without the indicated substances and their combinations. In the case of inhibition, the combination index (CI) was calculated and is indicated in the figure. Dashed lines in the bar graphs indicate the computed sum of the respective single substance treatments. 

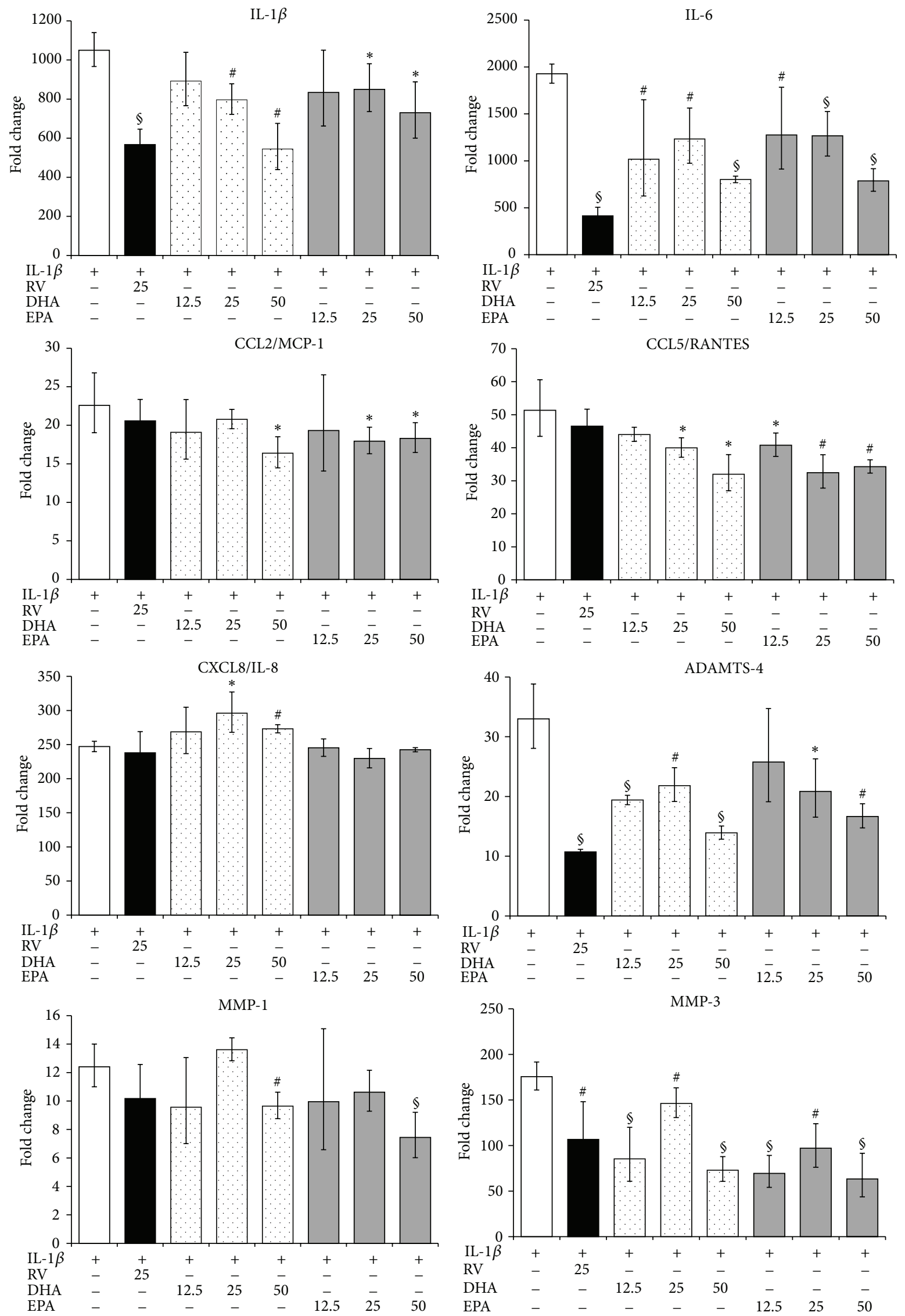

Figure 4: Continued. 

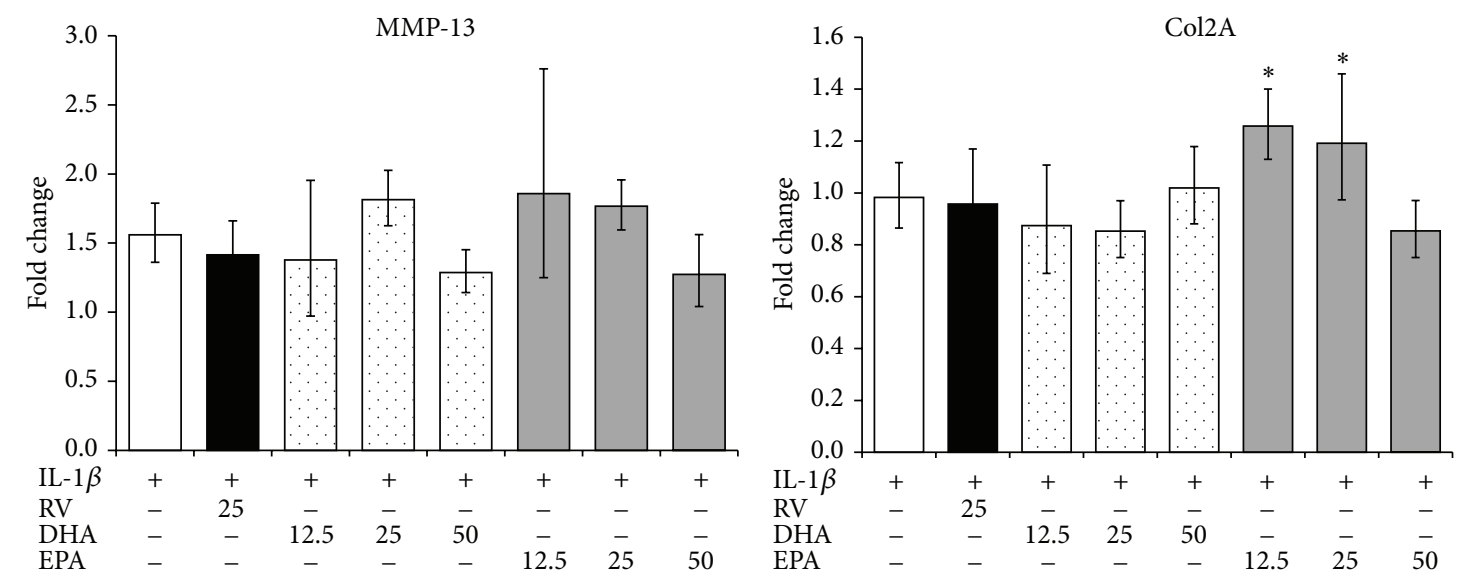

FIgURE 4: Modulation of gene expression in IL-1 $\beta$ activated human chondrocytes. Cells were activated with IL-1 $\beta$ with or without the indicated substances for $4 \mathrm{~h}$. Gene expression was quantified by RT-PCR and the data expressed as fold change compared to levels observed in unstimulated cells. ${ }^{*} p<0.05,{ }^{\#} p<0.01$, and ${ }^{\S} p<0.001$ (IL-1 $\beta$ only versus IL- $1 \beta+$ substance).

suggest that acute supplementation with EPA and DHA might transiently disturb homeostasis in PBLs in vitro and ex vivo, whereas during long-term supplementations cells no longer sense subtle homeostatic changes. Cytokines and chemokines critically determine macrophage differentiation and function [43]. In addition, the $\mathrm{T}_{h} 1$ and $\mathrm{T}_{\mathrm{h}} 2$ cell development is orchestrated by IL- 6 , IL-12, TNF- $\alpha$, and chemokines and their receptors [44]. Since immune cells sense, and respond to, the presence of RV and $\omega-3$ PUFAs by changes in cytokine and chemokine production, we hypothesize that these substances influence the differentiation of M2 macrophages and $\mathrm{T}_{\mathrm{h}} 2$ cells [45]. For instance, an increase of IL-6 is expected to favour the differentiation of M2 macrophages [46].

In this study we also show that $\omega$-3 PUFA modulated mRNA levels in human chondrocytes that were activated with the pathophysiological mediator IL- $1 \beta$. These cells are used as an appropriate in vitro model for chronic inflammation, which is a typical feature of osteoarthritis. Notably, expression levels of MMPs, ADAMTS-4, and interleukins were reduced. Similar data were obtained when chondrocytes were incubated with rose hip that also contained significant amounts of free fatty acids including EPA or DHA [47]. Other studies in bovine chondrocytes and cartilage explants have identified that long-term in vitro treatment of chondrocytes with conjugated linoleic acids or EPA mitigated the production of $\mathrm{PGE}_{2}$ and nitric oxide [48]. In bovine cartilage explants and chondrocytes, EPA affected ECM degradation, since it reduced glycosaminoglycan and collagen II release [49] and reduced gene expression of enzymes involved in OA [50]. Collectively, RV was less active on chondrocytes than $\omega-3$ PUFAs.

From these data we infer that RV and $\omega-3$ PUFAs modulate acute (in PBLs) and chronic inflammation (in chondrocytes) in different ways: RV mitigates early inflammatory events like the production of $\mathrm{PGE}_{2}$. $\omega$-3 PUFAs augment the amplitude and kinetics of inflammatory events in acute inflammation and its resolution. Yet, both substances diminish inflammatory processes during chronic inflammation.

\section{Conclusions}

$\omega$-3 PUFAs and RV differ in the regulation of acute inflammation in leukocytes, but they have common properties in modulating biochemical events related to chronic inflammation of chondrocytes.

\section{Conflict of Interests}

All authors are employees of DSM Nutritional Products, a leading manufacturer of vitamins and PUFAs.

\section{Authors' Contribution}

Joseph Schwager and Nathalie Richard contributed equally to this work. Joseph Schwager, Nathalie Richard, Christoph Riegger, and Norman Salem Jr. designed the experiments. Nathalie Richard performed the experiments. Joseph Schwager and Nathalie Richard analysed the data. Joseph Schwager has written the paper. All authors read and approved the paper.

\section{Acknowledgment}

The authors thank Daniel Raederstorff for the insightful comments.

\section{References}

[1] P. C. Calder, "n-3 Polyunsaturated fatty acids, inflammation, and inflammatory diseases," The American Journal of Clinical Nutrition, vol. 83, supplement 6, pp. 1505S-1519S, 2006.

[2] R. S. Chapkin, W. Kim, J. R. Lupton, and D. N. McMurray, "Dietary docosahexaenoic and eicosapentaenoic acid: emerging mediators of inflammation," Prostaglandins, Leukotrienes, and Essential Fatty Acids, vol. 81, no. 2-3, pp. 187-191, 2009.

[3] R. González, I. Ballester, R. López-Posadas et al., "Effects of flavonoids and other polyphenols on inflammation," Critical 
Reviews in Food Science and Nutrition, vol. 51, no. 4, pp. 331362, 2011.

[4] C. N. Serhan, N. Chiang, and T. E. Van Dyke, "Resolving inflammation: dual anti-inflammatory and pro-resolution lipid mediators," Nature Reviews Immunology, vol. 8, no. 5, pp. 349-361, 2008.

[5] M. Bouwens, O. Van De Rest, N. Dellschaft et al., "Fishoil supplementation induces antiinflammatory gene expression profiles in human blood mononuclear cells," The American Journal of Clinical Nutrition, vol. 90, no. 2, pp. 415-424, 2009.

[6] K. Kasuga, R. Yang, T. F. Porter et al., "Rapid appearance of resolvin precursors in inflammatory exudates: novel mechanisms in resolution," Journal of Immunology, vol. 181, no. 12, pp. 8677-8687, 2008.

[7] P. Kohli and B. D. Levy, "Resolvins and protectins: mediating solutions to inflammation," British Journal of Pharmacology, vol. 158, no. 4, pp. 960-971, 2009.

[8] A. Mullen, C. E. Loscher, and H. M. Roche, "Anti-inflammatory effects of EPA and DHA are dependent upon time and doseresponse elements associated with LPS stimulation in THP-1derived macrophages," The Journal of Nutritional Biochemistry, vol. 21, no. 5, pp. 444-450, 2010.

[9] V. Pallarès, D. Calay, L. Cedó et al., "Enhanced anti-inflammatory effect of resveratrol and EPA in treated endotoxinactivated RAW 264.7 macrophages," The British Journal of Nutrition, vol. 108, no. 9, pp. 1562-1573, 2012.

[10] M. M. Rahman, A. Bhattacharya, and G. Fernandes, "Docosahexaenoic acid is more potent inhibitor of osteoclast differentiation in RAW 264.7 cells than eicosapentaenoic acid," Journal of Cellular Physiology, vol. 214, no. 1, pp. 201-209, 2008.

[11] C. L. L. Saw, Y. Huang, and A.-N. Kong, "Synergistic antiinflammatory effects of low doses of curcumin in combination with polyunsaturated fatty acids: docosahexaenoic acid or eicosapentaenoic acid," Biochemical Pharmacology, vol. 79, no. 3, pp. 421-430, 2010.

[12] C. N. Serhan, S. Hong, K. Gronert et al., "Resolvins: a family of bioactive products of omega-3 fatty acid transformation circuits initiated by aspirin treatment that counter proinflammation signals," The Journal of Experimental Medicine, vol. 196, no. 8, pp. 1025-1037, 2002.

[13] I. Vedin, T. Cederholm, Y. F. Levi et al., "Effects of docosahexaenoic acid-rich n-3 fatty acid supplementation on cytokine release from blood mononuclear leukocytes: the OmegAD study," American Journal of Clinical Nutrition, vol. 87, no. 6, pp. 1616-1622, 2008.

[14] I. Vedin, T. Cederholm, Y. Freund-Levi et al., "Effects of DHArich n-3 fatty acid supplementation on gene expression in blood mononuclear leukocytes: the omegAD study," PLoS ONE, vol. 7, no. 4, Article ID e35425, 2012.

[15] S. M. Weldon, A. C. Mullen, C. E. Loscher, L. A. Hurley, and H. M. Roche, "Docosahexaenoic acid induces an anti-inflammatory profile in lipopolysaccharide-stimulated human THP-1 macrophages more effectively than eicosapentaenoic acid," The Journal of Nutritional Biochemistry, vol. 18, no. 4, pp. 250-258, 2007.

[16] C. M. Yates, P. C. Calder, and G. Ed Rainger, "Pharmacology and therapeutics of omega-3 polyunsaturated fatty acids in chronic inflammatory disease," Pharmacology \& Therapeutics, vol. 141, no. 3, pp. 272-282, 2014.

[17] P. C. Calder, N. Ahluwalia, F. Brouns et al., "Dietary factors and low-grade inflammation in relation to overweight and obesity,"
British Journal of Nutrition, vol. 106, supplement 3, pp. S5-S78, 2011.

[18] U. Švajger and M. Jeras, "Anti-inflammatory effects of resveratrol and its potential use in therapy of immune-mediated diseases," International Reviews of Immunology, vol. 31, no. 3, pp. 202-222, 2012.

[19] N. Richard, D. Porath, A. Radspieler, and J. Schwager, "Effects of resveratrol, piceatannol, tri-acetoxystilbene, and genistein on the inflammatory response of human peripheral blood leukocytes," Molecular Nutrition \& Food Research, vol. 49, no. 5, pp. 431-442, 2005.

[20] J. Schwager, U. Hoeller, S. Wolfram, and N. Richard, "Rose hip and its constituent galactolipids confer cartilage protection by modulating cytokine, and chemokine expression," BMC Complementary and Alternative Medicine, vol. 11, article 105, 2011.

[21] T.-C. Chou and P. Talalay, "Quantitative analysis of dose-effect relationships: the combined effects of multiple drugs or enzyme inhibitors," Advances in Enzyme Regulation, vol. 22, pp. 27-55, 1984.

[22] T. C. Chou and P. Talaly, "A simple generalized equation for the analysis of multiple inhibitions of Michaelis-Menten kinetic systems," The Journal of Biological Chemistry, vol. 252, no. 18, pp. 6438-6442, 1977.

[23] G. Pappa, J. Strathmann, M. Löwinger, H. Bartsch, and C. Gerhäuser, "Quantitative combination effects between sulforaphane and 3,3'-diindolylmethane on proliferation of human colon cancer cells in vitro," Carcinogenesis, vol. 28, no. 7, pp. 1471-1477, 2007.

[24] M. Baggiolini, "Chemokines and leukocyte traffic," Nature, vol. 392, no. 6676, pp. 565-568, 1998.

[25] H. Roca, Z. S. Varcos, S. Sud, M. J. Craig, and K. J. Pienta, "CCL2 and interleukin- 6 promote survival of human $\mathrm{CD}_{11 \mathrm{~b}^{+}}$peripheral blood mononuclear cells and induce M2-type macrophage polarization," The Journal of Biological Chemistry, vol. 284, no. 49, pp. 34342-34354, 2009.

[26] J. M. Wang, B. Sherry, M. J. Fivash, D. J. Kelvin, and J. J. Oppenheim, "Human recombinant macrophage inflammatory protein- $1 \alpha$ and $-\beta$ and monocyte chemotactic and activating factor utilize common and unique receptors on human monocytes," Journal of Immunology, vol. 150, no. 7, pp. 3022-3029, 1993.

[27] J. Seok, H. S. Warren, A. G. Cuenca et al., "Genomic responses in mouse models poorly mimic human inflammatory diseases," Proceedings of the National Academy of Sciences of the United States of America, vol. 110, no. 9, pp. 3507-3512, 2013.

[28] N. Alaaeddine, T. Olee, S. Hashimoto, L. Creighton-Achermann, and M. Lotz, "Production of the chemokine RANTES by articular chondrocytes and role in cartilage degradation," Arthritis and Rheumatism, vol. 44, no. 7, pp. 1633-1643, 2001.

[29] S. R. Goldring and M. B. Goldring, "The role of cytokines in cartilage matrix degeneration in osteoarthritis," Clinical Orthopaedics and Related Research, no. 427, supplement, pp. S27-S36, 2004.

[30] L. J. Sandell, X. Xing, C. Franz, S. Davies, L.-W. Chang, and D. Patra, "Exuberant expression of chemokine genes by adult human articular chondrocytes in response to IL-1 $\beta$," Osteoarthritis and Cartilage, vol. 16, no. 12, pp. 1560-1571, 2008. 
[31] K. Ashikawa, S. Majumdar, S. Banerjee, A. C. Bharti, S. Shishodia, and B. B. Aggarwal, "Piceatannol inhibits TNFinduced NF- $\kappa \mathrm{B}$ activation and NF- $\kappa \mathrm{B}$-mediated gene expression through suppression of $\mathrm{I} \kappa \mathrm{B} \alpha$ kinase and $\mathrm{p} 65$ phosphorylation," Journal of Immunology, vol. 169, no. 11, pp. 6490-6497, 2002.

[32] S.-H. Tsai, S.-Y. Lin-Shiau, and J.-K. Lin, "Suppression of nitric oxide synthase and the down-regulation of the activation of $\mathrm{NF} \kappa \mathrm{B}$ in macrophages by resveratrol," British Journal of Pharmacology, vol. 126, no. 3, pp. 673-680, 1999.

[33] P. C. Norris and E. A. Dennis, "Omega-3 fatty acids cause dramatic changes in TLR4 and purinergic eicosanoid signaling," Proceedings of the National Academy of Sciences of the United States of America, vol. 109, no. 22, pp. 8517-8522, 2012.

[34] K. Derecka, E. L. Sheldrick, D. C. Wathes, D. R. E. Abayasekara, and A. P. F. Flint, "A PPAR-independent pathway to PUFAinduced COX-2 expression," Molecular and Cellular Endocrinology, vol. 287, no. 1-2, pp. 65-71, 2008.

[35] P. J. Gillies, S. K. Bhatia, L. A. Belcher, D. B. Hannon, J. T. Thompson, and J. P. Vanden Heuvel, "Regulation of inflammatory and lipid metabolism genes by eicosapentaenoic acid-rich oil," Journal of Lipid Research, vol. 53, no. 8, pp. 1679-1689, 2012.

[36] B. Xue, Z. Yang, X. Wang, and H. Shi, "Omega-3 polyunsaturated fatty acids antagonize macrophage inflammation via activation of AMPK/SIRT1 pathway," PLoS ONE, vol. 7, no. 10, Article ID e45990, 2012.

[37] K. Fritsche, "Fatty acids as modulators of the immune response," Annual Review of Nutrition, vol. 26, pp. 45-73, 2006.

[38] J. W. C. Sijben and P. C. Calder, "Differential immunomodulation with long-chain n-3 PUFA in health and chronic disease," Proceedings of the Nutrition Society, vol. 66, no. 2, pp. 237-259, 2007.

[39] G. K. Pot, I. A. Brouwer, A. Enneman, G. T. Rijkers, E. Kampman, and A. Geelen, "No effect of fish oil supplementation on serum inflammatory markers and their interrelationships: a randomized controlled trial in healthy, middle-aged individuals," European Journal of Clinical Nutrition, vol. 63, no. 11, pp. 1353-1359, 2009.

[40] R. Gorjão, R. Verlengia, T. M. D. Lima et al., "Effect of docosahexaenoic acid-rich fish oil supplementation on human leukocyte function," Clinical Nutrition, vol. 25, no. 6, pp. 923938, 2006.

[41] J. M. Nauroth, Y. C. Liu, M. van Elswyk et al., "Docosahexaenoic acid (DHA) and docosapentaenoic acid (DPAn-6) algal oils reduce inflammatory mediators in human peripheral mononuclear cells in vitro and paw edema in vivo," Lipids, vol. 45 , no. 5 , pp. 375-384, 2010.

[42] M. C. W. Myhrstad, I. Narverud, V. H. Telle-Hansen et al., "Effect of the fat composition of a single high-fat meal on inflammatory markers in healthy young women," The British Journal of Nutrition, vol. 106, no. 12, pp. 1826-1835, 2011.

[43] A. Sica and A. Mantovani, "Macrophage plasticity and polarization: in vivo veritas," The Journal of Clinical Investigation, vol. 122, no. 3, pp. 787-795, 2012.

[44] F. Sallusto, A. Lanzavecchia, and C. R. MacKay, "Chemokines and chemokine receptors in T-cell priming and Th1/Th2- mediated responses," Immunology Today, vol. 19, no. 12, pp. 568-574, 1998.

[45] A. Jaudszus, M. Gruen, B. Watzl et al., "Evaluation of suppressive and pro-resolving effects of EPA and DHA in human primary monocytes and T-helper cells," Journal of Lipid Research, vol. 54, no. 4, pp. 923-935, 2013.
[46] J. Mauer, B. Chaurasia, J. Goldau et al., "Signaling by IL-6 promotes alternative activation of macrophages to limit endotoxemia and obesity-associated resistance to insulin," Nature Immunology, vol. 15, no. 5, pp. 423-430, 2014.

[47] J. Schwager, N. Richard, R. Schoop, and S. Wolfram, "A novel rose hip preparation with enhanced anti-inflammatory and chondroprotective effects," Mediators of Inflammation, vol. 2014, Article ID 105710, 10 pages, 2014.

[48] C.-L. Shen, D. M. Dunn, J. H. Henry, Y. Li, and B. A. Watkins, "Decreased production of inflammatory mediators in human osteoarthritic chondrocytes by conjugated linoleic acids," Lipids, vol. 39, no. 2, pp. 161-166, 2004.

[49] A. K. T. Wann, J. Mistry, E. J. Blain, A. T. Michael-Titus, and M. M. Knight, "Eicosapentaenoic acid and docosahexaenoic acid reduce interleukin- $1 \beta$-mediated cartilage degradation," Arthritis Research \& Therapy, vol. 12, no. 6, article R207, 2010.

[50] Z. Zainal, A. J. Longman, S. Hurst et al., "Relative efficacies of omega-3 polyunsaturated fatty acids in reducing expression of key proteins in a model system for studying osteoarthritis," Osteoarthritis and Cartilage/OARS, Osteoarthritis Research Society, vol. 17, no. 7, pp. 896-905, 2009. 

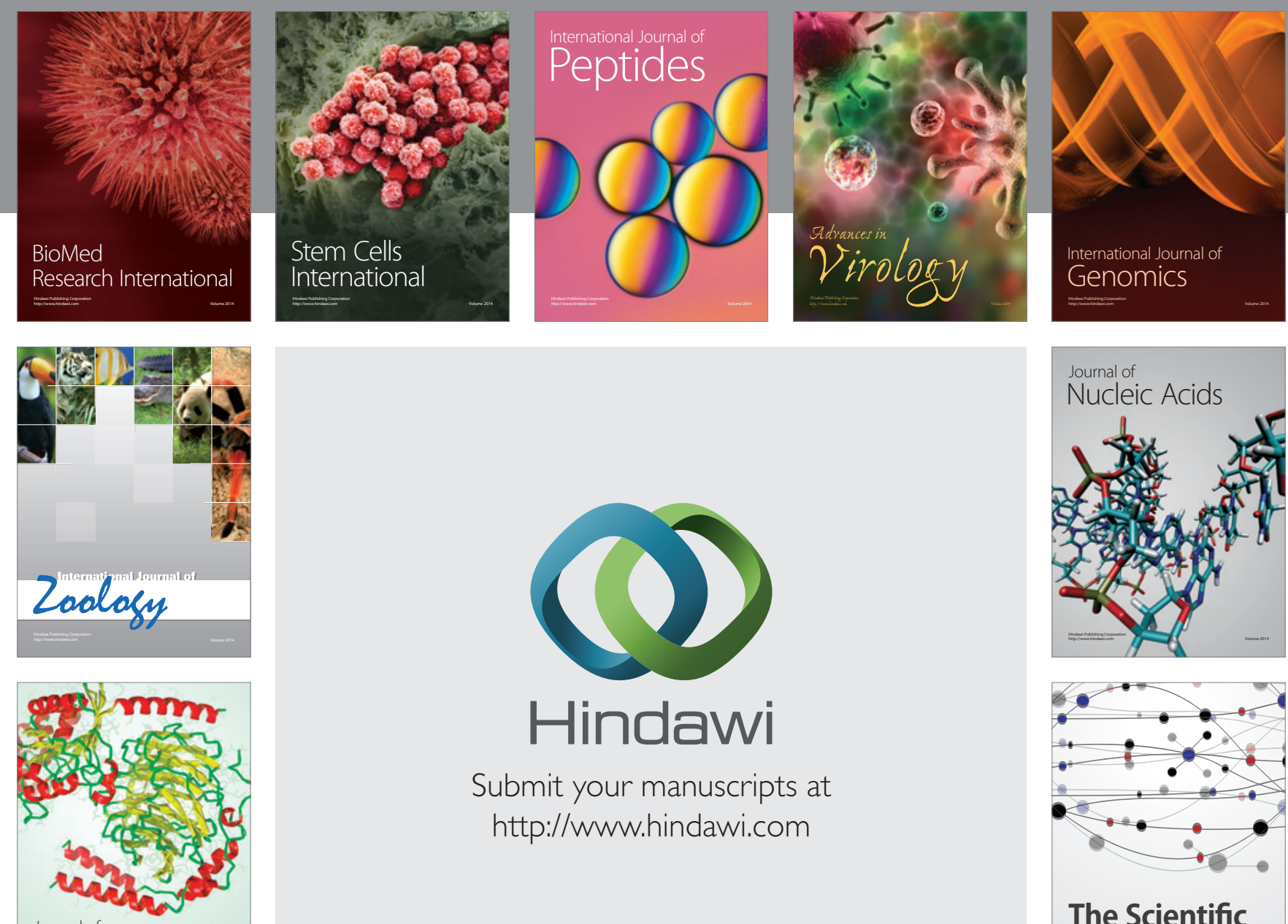

Submit your manuscripts at

http://www.hindawi.com

Journal of
Signal Transduction
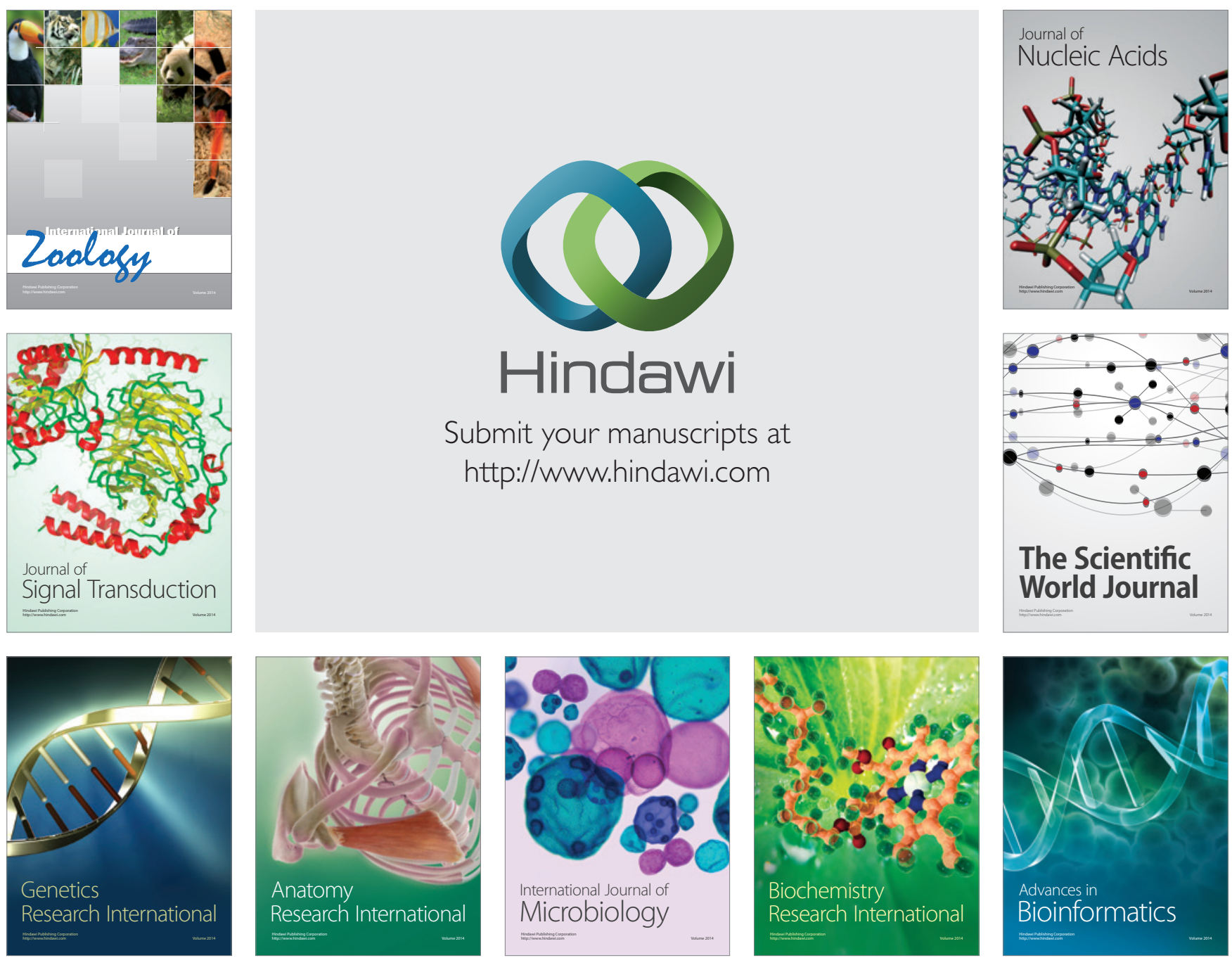

The Scientific World Journal
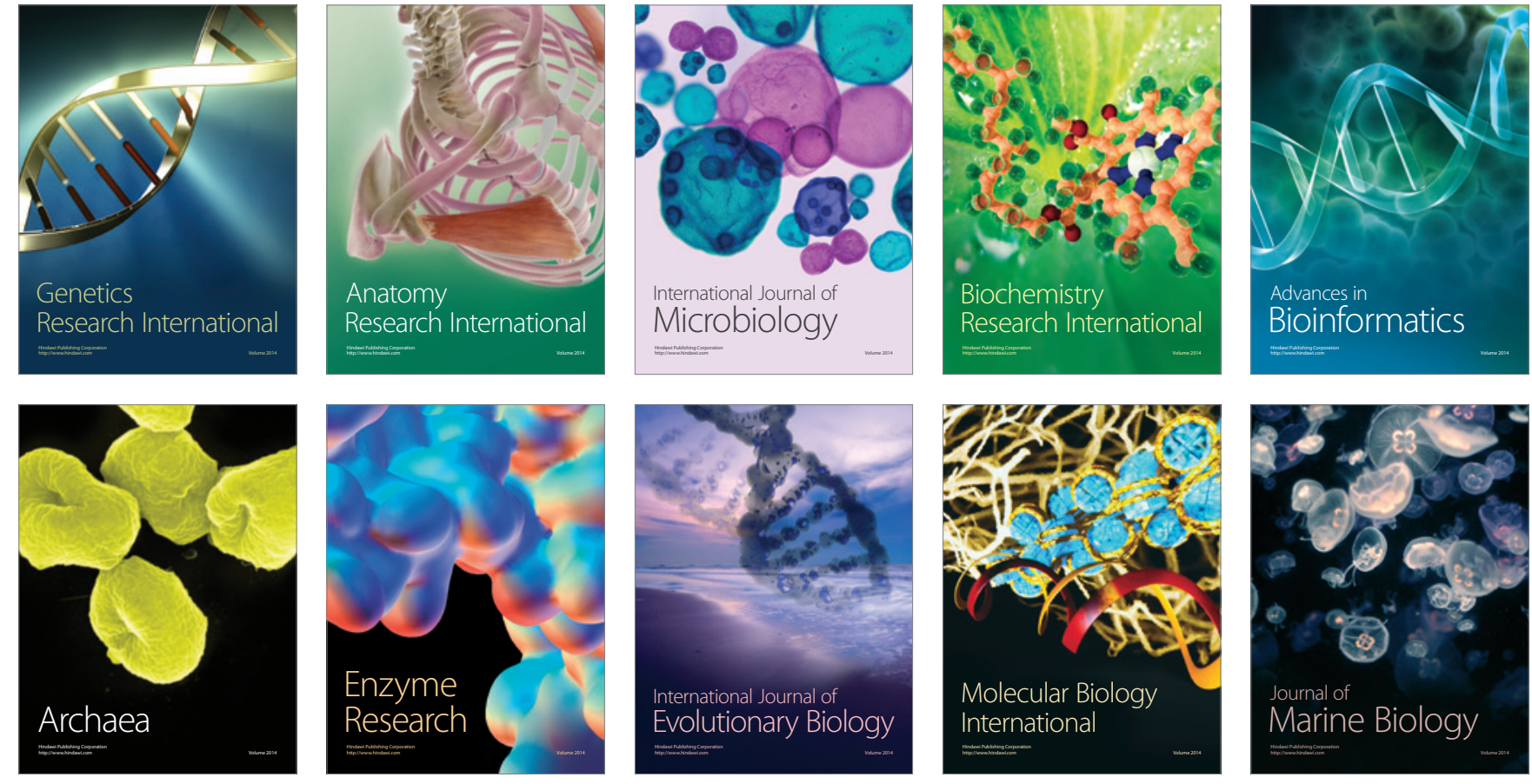